\title{
Multipole expansions in four-dimensional hyperspherical harmonics
}

\author{
A. V. Meremianin* \\ Max-Planck-Institute for the Physics of Complex Systems, \\ Nöthnitzer Str. 38, 01187, Dresden, Germany and \\ REC-010, Voronezh State University, 394006, Voronezh, Russia
}

(Dated: October 24, 2005)

\begin{abstract}
The technique of vector differentiation is applied to the problem of the derivation of multipole expansions in four-dimensional space. Explicit expressions for the multipole expansion of the function $r^{n} C_{j}(\hat{\mathbf{r}})$ with $\mathbf{r}=\mathbf{r}_{1}+\mathbf{r}_{2}$ are given in terms of tensor products of two hyperspherical harmonics depending on the unit vectors $\hat{\mathbf{r}}_{1}$ and $\hat{\mathbf{r}}_{2}$. The multipole decomposition of the function $\left(\mathbf{r}_{1} \cdot \mathbf{r}_{2}\right)^{n}$ is also derived. The proposed method can be easily generalised to the case of the space with dimensionality larger than four. Several explicit expressions for the four-dimensional ClebschGordan coefficients with particular values of parameters are presented in the closed form.
\end{abstract}

*Electronic address: avm@mpipks-dresden.mpg.de 


\section{INTRODUCTION}

The quantum angular momentum theory has proved to be a very efficient tool in many physical problems. Among the basis objects of that theory are the spherical harmonics which are the solution of the angular part of the Laplace equation. For spaces with dimensionality larger than three, the eigenfunctions of the angular part of the Laplacian are often called "hyperspherical harmonics" (HSH). From the point of view of quantum mechanics HSH are eigenfunctions of the total angular momentum operator. That explains why HSH have found wide use in the theory of quantum few-particle systems.

The theory of three-dimensional spherical harmonics is well developed since J.C. Maxwell's work on electromagnetic theory. In particular, the use of spherical harmonics allows one to simplify the calculation of various angular integrals. However, before such an integral can be calculated, one has to re-write the integrand functions in terms of spherical harmonics. The decomposition of a some function in terms of spherical harmonics is called the multipole expansion. There is a large number of known multipole expansion formulas for different functions in three-dimensional space, see e.g. [1, 2].

The expansion formulas in many-dimensional space are less known. The multipole expansion for the $\operatorname{exponential~function~} \exp (i \mathbf{a} \cdot \mathbf{b})$ has been derived in [3]. This exponential function is a scalar and its multipole decomposition is equivalent to the expansion in terms of Gegenbauer polynomials. In the paper [3] also the differential method for deriving the multipole expansions of scalar functions of the kind $f\left(\left|\mathbf{r}_{1}-\mathbf{r}_{2}\right|\right)$ has been developed.

Generally, the explicit expressions for the coefficients of multipole expansions (so-called "multipole coefficients") can be obtained using the orthogonality properties of spherical harmonics. This means that the multipole coefficients are determined by calculating the overlap integrals between the function to be expanded and the corresponding HSH. It is clear that in spaces with higher dimensions those overlap integrals will become more and more complicated.

The main goal of the present paper is to develop the differential technique for the derivation of multipole expansions. In the three-dimensional space such a technique is already known [4]. In the present paper, the multipole expansions in four-dimensional space are considered. The study of multipole expansions in four-dimensional space is motivated mainly by the fact that four-dimensional spherical harmonics represent the wave function of the 
hydrogen atom in momentum space [5]. Such harmonics are also used as Sturmian basis functions in many-centre Coulomb problems, see e.g. [6, 7]. We note also that the classical electrodynamics is built in the four-dimensional Minkowski space. Formally, this space corresponds to the space of vectors whose three (space) components are real and the fourth (time) component is purely imaginary. The rotations in Minkowski space are represented by the Lorentz group. The multipole expansion technique developed below remains the same also in Minkowski space.

As an example of the developed formalism, several expansion formulas are derived for tensor functions depending on the linear vector combination $\mathbf{r}_{1}+\mathbf{r}_{2}$. The proposed method can easily be generalised to the case of many-dimensional spaces. However, this requires the extensive study of the properties of corresponding Clebsch-Gordan coefficients which is not the subject of the present paper.

The paper is organised as follows. In Section II the general properties of four-dimensional HSH are discussed. Unlike three-dimensional spherical harmonics where there is only one set of their indices (and arguments) exist, HSH in four-dimensions can be parametrised in several ways. These are analysed in ref. [8]. Below we consider only two sets most important for practical applications. Keeping in mind the hydrogenic $O(4)$-symmetry, we denote them as spherical-type and parabolic-type HSH. In Section III the multipole expansion differential technique for four-dimensions HSH is developed. It is based on the rearrangement formula for the exponent $\exp (\mathbf{a} \cdot \nabla)$ where $\nabla$ is the gradient operator and $\mathbf{a}$ is an arbitrary vector in four-dimensional space. Although the expressions for the Clebsch-Gordan coefficients (CGC) of the $O(4)$ group are known (see e.g. $[9,10]$ ), the definitions used in common literature may differ in phase. Therefore, below the explicit expressions for CGC are considered. Analysis shows that for some particular values of indices, CGC can be written in a compact closed form. The corresponding expressions are given in Appendix A.

\section{HYPERSPHERICAL HARMONICS IN FOUR-DIMENSIONAL SPACE}

In this section the properties of irreducible tensors in four-dimensional space are analysed. The Cartesian components of the vector $\mathbf{r}$ in four-dimensional space we denote as $\left(x, y, z, z_{0}\right)$. We will also use the set of hyperspherical coordinates $r, \theta_{0}, \theta, \phi$ which are connected to 
Cartesian coordinates by means of identities

$$
\begin{aligned}
x & =r \sin \theta_{0} \sin \theta \cos \phi, \\
y & =r \sin \theta_{0} \sin \theta \sin \phi, \\
z & =r \sin \theta_{0} \cos \theta, \\
z_{0} & =r \cos \theta_{0} .
\end{aligned}
$$

As is seen $\theta_{0}$ is the angle between the $x_{0}$-axis of the coordinate frame and the vector $\mathbf{r}$. (We note that in Minkowski space the length is defined as $r^{2}=z_{0}^{2}-x^{2}-y^{2}-z^{2}$ and the replacements $\sin \theta_{0} \rightarrow i \sinh \theta_{0}$ and $\cos \theta_{0} \rightarrow \cosh \theta_{0}$ should be made.) The inverse relations are

$$
\begin{aligned}
r^{2} & =x^{2}+y^{2}+z^{2}+z_{0}^{2}, \\
\theta_{0} & =\arccos \frac{z_{0}}{r}, \\
\theta & =\arctan \frac{\sqrt{x^{2}+y^{2}}}{z}, \\
\phi & =\arctan \frac{y}{x} .
\end{aligned}
$$

(The inverse relations in Minkowski space can be derived similarly.)

By definition, the irreducible tensor is a traceless tensor, symmetric with respect to the interchange of any pair of its indices. The number of different components of the rank- $j$ irreducible tensor in four-dimensional space gives the number $(j+1)^{2}$. This means that the irreducible tensor can be labelled with two indices each of which runs from 0 to $j$. Tensor products $C_{j, \mu, \nu}(\hat{\mathbf{r}})$ will be denoted as parabolic-type set of HSH. There exists another possibility of choosing the set of tensor indices. Namely, one can use indices $\lambda$ and $\alpha$ so that $\lambda$ varies from 0 to $j$ and $\alpha$ runs from $-\lambda$ to $\lambda$. The total number of all possible combinations of indices $\lambda$ and $\alpha$ remains, of course, equal to $(j+1)^{2}$. The set of functions $C_{j, \lambda, \alpha}(\hat{\mathbf{r}})$ will be referred to as spherical-type HSH.

It is easy to see that the irreducible tensor product of $n$ vectors $\mathbf{r}$ satisfies the Laplace equation,

$$
\begin{aligned}
& \Delta\{\mathbf{r}\}_{j, \mu, \nu}=0 \\
& \Delta=\frac{\partial^{2}}{\partial x^{2}}+\frac{\partial^{2}}{\partial y^{2}}+\frac{\partial^{2}}{\partial z^{2}}+\frac{\partial^{2}}{\partial z_{0}^{2}} .
\end{aligned}
$$

Indeed, the tensor $\{\mathbf{r}\}_{j, \mu, \nu}$ is a homogeneous polynomial of the order $j$ with respect to the components $r_{1, \mu, \nu}$. The action of the scalar Laplace operator $\Delta$ on such a polynomial 
decreases its order by two. However, the result of this action should still be an irreducible tensor of the same rank $j$ because the tensor equality must contain only tensors of equal ranks. Thus, the above statement in proved by contradiction. We note also that the tensor product $\{\mathbf{r}\}_{j, \mu, \nu}=r^{j}\{\hat{\mathbf{r}}\}_{j, \mu, \nu}$ satisfies the boundary condition

$$
\lim _{r \rightarrow 0}\{\mathbf{r}\}_{j, \mu, \nu} \rightarrow 0
$$

i.e. it vanishes at the origin. Another tensor solution of the four-dimensional Laplace equation which is divergent at the origin has the form $r^{-j-2}\{\hat{\mathbf{r}}\}_{j, \mu, \nu}$. Clearly, the tensor product of unit vectors $\hat{\mathbf{r}},\{\hat{\mathbf{r}}\}_{j, \mu, \nu}$ is independent of the hyperradius $r$, and, hence, it is the function of hyperangles. Therefore, the tensor product $\{\hat{\mathbf{r}}\}_{j, \mu, \nu}$ coincides with HSH up to normalisation factor. (The same is true also for the set of indices $j, \lambda, \alpha$.)

Below, we consider two possible parametrisations for the four-dimensional HSH. In sec. II A the parabolic-type set of HSH is introduced. This set is particularly convenient for the calculations since the corresponding Clebsch-Gordan coefficients have simple form. The spherical-type HSH are discussed in sec. II B. Such harmonics describe the wave functions of the hydrogen atom in momentum space labelled with spherical quantum numbers $n, l, m$. The Clebsch-Gordan coefficients for spherical-type HSH have more complicated structure comparing to that of the parabolic-type HSH.

\section{A. Parabolic-type spherical harmonics}

Now let us introduce the hyperspherical components of the vector $\mathbf{r}$ according to the relations

$$
\begin{aligned}
& r_{ \pm \frac{1}{2}, \pm \frac{1}{2}}=\frac{1}{\sqrt{2}}\left(z_{0} \mp i z\right), \\
& r_{ \pm \frac{1}{2}, \mp \frac{1}{2}}=-\frac{i}{\sqrt{2}}(x \mp i y) .
\end{aligned}
$$

From these equations it follows that

$$
r_{\mu, \nu}^{*}=(-1)^{\mu-\nu} r_{-\mu,-\nu}, \quad \mu, \nu=-\frac{1}{2}, \frac{1}{2} .
$$

The square length of $\mathbf{r}$ in terms of these components has the form

$$
r^{2}=\sum_{\mu, \nu=-\frac{1}{2}}^{\frac{1}{2}} r_{\mu, \nu} r_{\mu, \nu}^{*}=\sum_{\mu, \nu}(-1)^{\mu-\nu} r_{\mu, \nu} r_{-\mu,-\nu}=x^{2}+y^{2}+z^{2}+z_{0}^{2} .
$$


The hyperspherical components of $\mathbf{r}$ are connected to the hyperangles as

$$
\begin{aligned}
& r_{ \pm \frac{1}{2}, \pm \frac{1}{2}}=\frac{r}{\sqrt{2}}\left(\cos \theta_{0} \mp i \sin \theta_{0} \cos \theta\right), \\
& r_{ \pm \frac{1}{2}, \mp \frac{1}{2}}= \pm i \frac{r}{\sqrt{2}} \sin \theta_{0} \sin \theta e^{\mp i \phi} .
\end{aligned}
$$

These equation can be re-written in terms of three-dimensional finite rotation matrices $U_{\mu \nu}^{\frac{1}{2}}$ parametrised by the direction of rotation and rotational angles [1],

$$
r_{\mu, \nu}=\frac{r}{\sqrt{2}} U_{\mu, \nu}^{1 / 2}\left(2 \theta_{0}, \theta, \phi\right) .
$$

We define the parabolic-type (or $H$-harmonics) spherical harmonics $H_{j, \mu, \nu}(\hat{\mathbf{r}})$ according to the relation

$$
\{\hat{\mathbf{r}}\}_{j, \mu, \nu}=\frac{1}{2^{j / 2}} H_{j, \mu, \nu}(\hat{\mathbf{r}})=\frac{1}{2^{j / 2}} U_{\mu, \nu}^{j / 2}\left(2 \theta_{0}, \theta, \phi\right),
$$

where $U_{\mu, \nu}^{j / 2}$ are elements of the three-dimensional finite rotation matrix. Written in terms of Euler angles these matrix elements are called Wigner's $D$-functions.

Let us consider $H$-harmonics for some specific values of its arguments and indices. The evaluation of the harmonics $H_{j, \mu, \nu}\left(\mathbf{e}_{z}\right)$ depending on the unit vector $\mathbf{e}_{0}$ with components $\mathbf{e}_{0}=(0,0,0,1)$ can easily be performed noting that its spherical coordinates are $r=1$, $\theta_{0}=\theta=\phi=0$. For this case we have $[1]$,

$$
H_{j, \mu, \nu}\left(\mathbf{e}_{z_{0}}\right)=U_{\mu, \nu}^{j / 2}(0,0,0)=\delta_{\mu, \nu}
$$

Note that $\mathbf{e}_{0}$ is directed along the $z_{0}$-axis of the coordinate frame.

The scalar product of two $H$-harmonics is defined as

$$
\left(H_{j}(\hat{\mathbf{a}}) \cdot H_{j}(\hat{\mathbf{b}})\right)=\sum_{\mu, \nu=-j / 2}^{j / 2}(-1)^{\mu-\nu} H_{j, \mu, \nu}(\hat{\mathbf{a}}) H_{j,-\mu,-\nu}(\hat{\mathbf{a}})=C_{j}^{1}(\hat{\mathbf{a}} \cdot \hat{\mathbf{b}}),
$$

where $C_{j}^{1}(\hat{\mathbf{a}} \cdot \hat{\mathbf{b}})$ is the Gegenbauer polynomial. The expansion formula for the product of two hyperspherical harmonics $H_{j, \mu, \nu}$ can be derived based on properties of the $3 d$-finite rotation matrices $U_{\mu, \nu}^{j / 2}$,

$$
H_{j_{1}, \mu_{1}, \nu_{1}}(\hat{\mathbf{r}}) H_{j_{2}, \mu_{2}, \nu_{2}}(\hat{\mathbf{r}})=\sum_{j=\left|j_{1}-j_{2}\right|}^{j_{1}+j_{2}} H_{j_{1} \mu_{1} \nu_{1} ; j_{2} \mu_{2} \nu_{2}}^{j \mu \nu} H_{j, \mu, \nu}(\hat{\mathbf{r}}),
$$

where $H_{j_{1} \mu_{1} \nu_{1} ; j_{2} \mu_{2} \nu_{2}}^{j \mu \nu}$ are the "parabolic-type" CGC for the $O(4)$ group. From the definition (10) of $H$-harmonics as $3 d$ finite rotation matrices it follows that H-type CGC are simply products of two CGC of the $O(3)$ group

$$
H_{j_{1} \mu_{1} \nu_{1} ; j_{2} \mu_{2} \nu_{2}}^{j, \mu, \nu}=C_{\left(j_{1} / 2\right) \mu_{1}\left(j_{2} / 2\right) \nu_{2}}^{(j / 2) \mu} C_{\left(j_{1} / 2\right) \nu_{1}\left(j_{2} / 2\right) \nu_{2}}^{(j / 2) \nu} .
$$


This expression for CGC directly follows from the definition of spherical harmonics as the elements of the finite rotation matrix in $3 d$-space. According to the above equations, the CGC are nonzero only if the total momentum $j$ takes values We will not discuss further properties of coefficients $H_{l \mu \mu^{\prime} ; l^{\prime} \nu \nu^{\prime}}^{j \alpha \alpha^{\prime}}$, such as orthogonality etc, since they can be easily deduced from the properties of conventional CGC in $3 d$-space.

From the expression (14) for CGC one can deduce the triangle rule for the momenta $j_{1}, j_{2}, j$. Namely, the momentum $j$ can take the values $\left|j_{1}-j_{2}\right|,\left|j_{1}-j_{2}\right|+2, \ldots, j_{1}+j_{2}$. Thus, the sum $j_{1}+j_{2}+j_{3}$ is always an even number. In particular, for $j_{1}=j_{2}$ we have that $j=0,2,4 \ldots,\left(2 j_{1}\right)$.

The tensor product of two spherical harmonics we will denote as $\left\{H_{j_{1}}(\hat{\mathbf{a}}) \otimes H_{j_{2}}(\hat{\mathbf{b}})\right\}_{j}$ and it is defined by the equation

$$
\left\{H_{j_{1}}(\hat{\mathbf{a}}) \otimes H_{j_{2}}(\hat{\mathbf{b}})\right\}_{j, \mu, \nu}=\sum_{\mu_{1}, \nu_{1}=-j_{1} / 2}^{j_{1} / 2} \sum_{\mu_{2}, \nu_{2}=-j_{2} / 2}^{j_{2} / 2} H_{j_{1} \mu_{1} \nu_{1} ; j_{2} \mu_{2} \nu_{2}}^{j, \mu, \nu} H_{j_{1}, \mu_{1}, \nu_{1}}(\hat{\mathbf{a}}) H_{j_{2}, \mu_{2}, \nu_{2}}(\hat{\mathbf{b}}) .
$$

Below, we will label the tensor products and spherical harmonics only with their ranks unless one needs to derive explicit expressions. The bipolar harmonics of the zero rank are connected with the scalar product of spherical harmonics as

$$
\left\{H_{l}(\hat{\mathbf{a}}) \otimes H_{l}(\hat{\mathbf{b}})\right\}_{0}=\frac{1}{l+1}\left(H_{l}(\hat{\mathbf{a}}) \cdot H_{l}(\hat{\mathbf{b}})\right) .
$$

For two equal vectors $\hat{\mathbf{a}}=\hat{\mathbf{b}}$ this equation becomes

$$
\left\{H_{l}(\hat{\mathbf{a}}) \otimes H_{l}(\hat{\mathbf{a}})\right\}_{0}=\frac{1}{l+1}\left(H_{l}(\hat{\mathbf{a}}) \cdot H_{l}(\hat{\mathbf{a}})\right)=1 .
$$

\section{B. Spherical-type spherical harmonics}

The explicit expression for the spherical-type HSH can be derived using the parametrisation of the $3 d$ finite rotation matrix in terms of the rotation angle and the rotation axis,

$$
U_{\mu, \nu}^{l}\left(2 \theta_{0}, \theta, \phi\right)=\sum_{\lambda=0}^{2 l} \sum_{\alpha=-\lambda}^{\lambda}(-i)^{\lambda} \frac{2 \lambda+1}{2 l+1} C_{l \mu \lambda \alpha}^{l \nu} \chi_{\lambda}^{l}\left(2 \theta_{0}\right) C_{\lambda \alpha}(\theta, \phi),
$$

where $C_{\lambda \alpha}$ are modified spherical harmonics connected to the usual $3 d$-spherical harmonics by $C_{\lambda \alpha}=\sqrt{4 \pi /(2 \lambda+1)} Y_{\lambda \alpha}$; The generalised characters of the $O(3)$ rotation group $\chi_{\lambda}^{l}$ can be expressed in terms of Gegenbauer polynomials [1],

$$
\chi_{\lambda}^{l}\left(2 \theta_{0}\right)=(2 \lambda) ! ! \sqrt{2 l+1} \sqrt{\frac{(2 l-\lambda) !}{(2 l+\lambda+1) !}}\left(\sin \theta_{0}\right)^{\lambda} C_{2 l-\lambda}^{\lambda+1}\left(\cos \theta_{0}\right) .
$$


We introduce the set of spherical-type ( $C$-harmonics) HSH $C_{j, \lambda, \mu}$ by connecting them to $H$-harmonics as

$$
C_{j \lambda \mu}(\hat{\mathbf{r}})=\sqrt{\frac{2 \lambda+1}{j+1}} \sum_{\mu, \nu=-j / 2}^{j / 2} C_{(j / 2) \nu \lambda \alpha}^{(j / 2) \mu} H_{j, \mu, \nu}(\hat{\mathbf{r}}),
$$

where $\lambda=0,1, \ldots j$, and $\alpha=-\lambda,-\lambda+1, \ldots \lambda$. The inverse relation can be derived using the orthogonality of $3 d \mathrm{CGC}$

$$
H_{j, \mu, \nu}(\hat{\mathbf{r}})=\sum_{\lambda=0}^{j} \sum_{\alpha=-\lambda}^{\lambda} \sqrt{\frac{2 \lambda+1}{j+1}} C_{(j / 2) \nu \lambda \alpha}^{(j / 2) \mu} C_{j, \lambda, \alpha}(\hat{\mathbf{r}}) .
$$

The explicit expression for $C$-harmonics can be obtained from (18) and it is

$$
C_{j, \lambda, \alpha}(\hat{\mathbf{r}})=(-i)^{\lambda} \sqrt{\frac{2 \lambda+1}{j+1}} \chi_{\lambda}^{j / 2}\left(2 \theta_{0}\right) C_{\lambda \alpha}(\theta, \phi) .
$$

From this equation follows the formula for complex conjugated harmonics

$$
C_{j, \lambda, \alpha}^{*}(\hat{\mathbf{r}})=(-1)^{\lambda+\alpha} C_{j, \lambda,-\alpha}(\hat{\mathbf{r}})
$$

The connection of $C$-harmonics with tensor products of vectors is the same as for $H$ harmonics which is given by eq. (10). The scalar product of two $C$-harmonics is defined similarly to the above eq. (12),

$$
\left(C_{j}(\hat{\mathbf{a}}) \cdot C_{j}(\hat{\mathbf{b}})\right)=\sum_{\lambda, \alpha} C_{j, \lambda, \alpha}^{*}(\hat{\mathbf{a}}) C_{j, \lambda, \alpha}(\hat{\mathbf{b}})=\sum_{\lambda, \alpha}(-1)^{\lambda+\alpha} C_{j, \lambda, \alpha}(\hat{\mathbf{a}}) C_{j, \lambda,-\alpha}(\hat{\mathbf{b}})=C_{j}^{1}(\hat{\mathbf{a}} \cdot \hat{\mathbf{b}}) .
$$

We note also the expression for $C_{j}\left(\mathbf{e}_{0}\right)$ which can be derived from eq. (20),

$$
C_{j, \lambda, \alpha}\left(\mathbf{e}_{0}\right)=(-i)^{\lambda} \sqrt{\frac{2 \lambda+1}{j+1}} \chi_{\lambda}^{j / 2}(0) C_{\lambda \alpha}(0,0)=\sqrt{j+1} \delta_{\lambda, 0} \delta_{\alpha, 0},
$$

where we have used the property of the generalised characters [1]

$$
\chi_{\lambda}^{j / 2}(0)=(j+1) \delta_{\lambda, 0}
$$

Now we have to establish the expression for CGC for the set of $C$-harmonics. We define those coefficients by considering the expansion of the product of two $C$-harmonics

$$
C_{j_{1}, \lambda_{1}, \alpha_{1}}(\hat{\mathbf{r}}) C_{j_{2}, \lambda_{2}, \alpha_{2}}(\hat{\mathbf{r}})=\sum_{j \lambda \alpha} C_{j_{1} \lambda_{1} \alpha_{1} ; j_{2} \lambda_{2} \alpha_{2}}^{j, \lambda} C_{j, \lambda, \alpha}(\hat{\mathbf{r}})
$$

CGC in this equation can be calculated by expressing each $C$-harmonic in terms of $H$ harmonics according to the decomposition (20). At this stage the CGC for $H$-harmonics 
will occur. However, these are already known, see eq. (14). After somewhat lengthy manipulations with $3 d$ CGC one arrives at the expression

$$
C_{j_{1} \lambda_{1} \alpha_{1} ; j_{2} \lambda_{2} \alpha_{2}}^{j, \lambda, \alpha}=(j+1) \sqrt{\left(2 \lambda_{1}+1\right)\left(2 \lambda_{2}+1\right)} C_{\lambda_{1} \alpha_{1} \lambda_{2} \alpha_{2}}^{\lambda \alpha}\left\{\begin{array}{ccc}
\frac{j_{1}}{2} & \frac{j_{2}}{2} & \frac{j}{2} \\
\frac{j_{1}}{2} & \frac{j_{2}}{2} & \frac{j}{2} \\
\lambda_{1} & \lambda_{2} & \lambda
\end{array}\right\}
$$

where the table in curly brackets is the $9 j$-coefficient of $3 d$-space. The $9 j$-coefficient with two identical rows in nonzero only if the sum of its indices is an even number [1]. Note, that the sum $j_{1}+j_{2}+j$ is always an even number. Hence, the $C$-type CGC is non-zero only if the combination $\lambda_{1}+\lambda_{2}+\lambda$ is an even number. Thus, the parameter $\lambda$ can be $\left|\lambda_{1}-\lambda_{2}\right|,\left|\lambda_{2}-\lambda_{2}\right|+2, \ldots, \lambda_{1}+\lambda_{2}$.

The bipolar harmonics of $C$-type are defined similarly to the $H$-case (15),

$$
\left\{C_{l}(\hat{\mathbf{a}}) \otimes C_{l^{\prime}}(\hat{\mathbf{b}})\right\}_{j, \lambda, \alpha}=\sum_{\lambda, \lambda^{\prime}, \alpha, \alpha^{\prime}} C_{l \lambda \alpha^{\prime} ; l^{\prime} \beta \beta^{\prime}}^{j \lambda \alpha} C_{l, \alpha, \alpha^{\prime}}(\hat{\mathbf{a}}) C_{l^{\prime}, \beta, \beta^{\prime}}(\hat{\mathbf{b}}) .
$$

The expressions (16), (17) for the scalar product of $H$-harmonics remain valid after the replacement $H \leftrightarrow C$.

We have to analyse the properties of the $C$-type CGC. Clearly, they follow from properties of the $3 d$ CGC and $9 j$-symbols. For example, the orthogonality relation of $4 d$ CGC has the form

$$
\sum_{\lambda_{1}, \lambda_{2}, \alpha_{1}, \alpha_{2}} C_{j_{1} \lambda_{1} \alpha_{1} ; j_{2} \lambda_{2} \alpha_{2}}^{j \lambda \alpha} C_{j_{1} \lambda_{1} \alpha_{1} ; j_{2} \lambda_{2} \alpha_{2}}^{j^{\prime} \lambda^{\prime} \alpha^{\prime}}=\delta_{j, j^{\prime}} \delta_{\lambda, \lambda^{\prime}} \delta_{\alpha, \alpha^{\prime}}
$$

The exchange symmetry of $4 d$ CGC is

$$
C_{j_{1} \lambda_{1} \alpha_{1} ; j_{2} \lambda_{2} \alpha_{2}}^{j \lambda \alpha}=(-1)^{j_{1}+j_{2}+j} C_{j_{2} \lambda_{2} \alpha_{2} ; j_{1} \lambda_{1} \alpha_{1}}^{j \lambda \alpha}
$$

We note that the above identities hold for both, $C$ - and $H$-types of CGC. We present also one more symmetry relation valid for $C$-type CGC

$$
C_{j_{1} \lambda_{1} \alpha_{1} ; j_{2} \lambda_{2} \alpha_{2}}^{j \lambda \alpha}=(-1)^{j_{1}+j_{2}+j+\lambda_{2}+\alpha_{2}} \frac{j+1}{j_{1}+1} C_{j \lambda \alpha ; j_{2} \lambda_{2}-\alpha_{2}}^{j_{1} \lambda_{1} \alpha_{1}}
$$

At some particular values of its parameters the $C$-type CGC may be written in closed form. Clearly, this happens at $j_{1}=0$ where

$$
C_{000 ; j_{2} \lambda_{2} \alpha_{2}}^{j \lambda \alpha}=\delta_{j, j_{2}} \delta_{\lambda, \lambda_{2}} \delta_{\alpha, \alpha_{2}}
$$


For zero projections, $C$-type CGC also evaluates to a closed form,

$$
C_{j_{1} 00 ; j_{2} 00}^{j 00}=\sqrt{\frac{j+1}{\left(j_{1}+1\right)\left(j_{2}+1\right)}} .
$$

More explicit expressions for $C$-type CGC are given in Appendix A.

We have to consider also the re-coupling coefficients in $4 d$-space. These re-coupling coefficients can depend only upon the ranks of tensors but not on their projection indices. Therefore, all tensor identities are covariant, i.e. they are equally valid for $H$ - and $C$-type tensor components. As an example, we consider the re-coupling relation

$$
\begin{aligned}
\left\{\left\{P_{a} \otimes Q_{b}\right\}_{c} \otimes\left\{R_{d} \otimes S_{e}\right\}_{f}\right\}_{k}=\sum_{g h}(c+1)(f+1)(g+1)(h+1) & \\
& \times\left\{\left\{P_{a} \otimes R_{d}\right\}_{g} \otimes\left\{Q_{b} \otimes S_{e}\right\}_{h}\right\}_{k}\left[\begin{array}{ccc}
a & b & c \\
d & e & f \\
g & h & k
\end{array}\right],
\end{aligned}
$$

where the table in square brackets is the $9 j$-symbol in $4 d$-space. Its calculation is more simple when using $H$-type CGC. Omitting the details of derivations we present only the result,

$$
\left[\begin{array}{lll}
a & b & c \\
d & e & f \\
g & h & k
\end{array}\right]=\left\{\begin{array}{ccc}
\frac{a}{2} & \frac{b}{2} & \frac{c}{2} \\
\frac{d}{2} & \frac{e}{2} & \frac{f}{2} \\
\frac{g}{2} & \frac{h}{2} & \frac{k}{2}
\end{array}\right\}^{2}
$$

As is seen, the irreducible tensor in $4 d$-space may be considered as "double-tensors" in $3 d$-space.

Finally, we note that HSH are orthogonal,

$$
\begin{array}{r}
\int_{S} C_{j, \lambda, \alpha}(\hat{\mathbf{r}}) C_{j^{\prime}, \lambda^{\prime}, \alpha^{\prime}}^{*}(\hat{\mathbf{r}}) d \Omega=\int_{0}^{\pi} \sin ^{2} \theta_{0} d \theta_{0} \int_{0}^{\pi} \sin \theta d \theta \int_{0}^{2 \pi} d \phi C_{j, \lambda, \alpha}\left(\theta_{0}, \theta, \phi\right) C_{j^{\prime}, \lambda^{\prime}, \alpha^{\prime}}^{*}\left(\theta_{0}, \theta, \phi\right) \\
=\frac{8 \pi^{2}}{j+1} \delta_{j, j^{\prime}} \delta_{\lambda, \lambda^{\prime}} \delta_{\alpha, \alpha^{\prime}}, \quad(36)
\end{array}
$$

where the integration is performed over the four-dimensional hypersphere $S$. Often, HSH normalised to unity are used

$$
Y_{j, \lambda, \alpha}(\hat{\mathbf{r}})=\frac{1}{2 \pi} \sqrt{\frac{j+1}{2}} C_{j, \lambda, \alpha}(\hat{\mathbf{r}}) .
$$

The orthogonality identity holds also for $H$-type spherical harmonics,

$$
\int_{S} H_{j, \mu, \nu}(\hat{\mathbf{r}}) H_{j^{\prime}, \mu^{\prime}, \nu^{\prime}}^{*}(\hat{\mathbf{r}}) d \Omega=\frac{8 \pi^{2}}{j+1} \delta_{j, j^{\prime}} \delta_{\mu, \mu^{\prime}} \delta_{\nu, \nu^{\prime}}
$$




\section{THE MULTIPOLE EXPANSIONS IN HYPERSPHERICAL HARMONICS}

In this section the differential formalism for the derivation of multipole expansions in four-dimensional space is developed. The general formulas are derived in Sec. III A. In Sec. III B we consider the multipole expansions of the function $|\mathbf{a}+\mathbf{r}|^{n} C_{j}(\hat{\mathbf{b}})$ where $\hat{\mathbf{b}}$ is the unit vector directed along the vector sum $(\mathbf{a}+\mathbf{r})$.

\section{A. The differential multipole expansion formula}

We start with the conventional Taylor expansion formula in many-dimensional space:

$$
f(\mathbf{a}+\mathbf{r})=\sum_{l=0}^{\infty} \frac{1}{l !}(\mathbf{a} \cdot \nabla)^{l} f(\mathbf{r})
$$

It can be re-written in the simple symbolic form,

$$
f(\mathbf{a}+\mathbf{r})=e^{(\mathbf{a} \cdot \nabla)} f(\mathbf{r}) .
$$

We have to present this formula in a way suitable for the derivation of multipole expansions of the function $f(\mathbf{r})$. This can be done similarly to the three-dimensional case considered in [4]. Namely, one has to use the multipole expansion for the operator exponent $\exp (\mathbf{a} \cdot \nabla)$. Before doing so, we write the multipole expansion of the exponential scalar product in four-dimensional space [3],

$$
e^{\mathbf{a} \cdot \mathbf{r}}=\sum_{l=0}^{\infty} \frac{i^{l+1}}{a r} 2(l+1) J_{l+1}(-i a r) C_{l}^{1}(\hat{\mathbf{a}} \cdot \hat{\mathbf{r}}) .
$$

The Bessel function $J_{l+1}(i a r)$ can be written in terms of a hypergeometric function [11] as,

$$
J_{l+1}(-i x)=\frac{(-i x)^{l+1}}{2^{l+1}(l+1) !}{ }_{0} F_{1}\left(l+2 ; x^{2} / 4\right) .
$$

This formula allows one to re-write the decomposition (41) in the form

$$
e^{\mathbf{a} \cdot \mathbf{r}}=\sum_{l=0}^{\infty} \frac{a^{l} r^{l}}{2^{l} l !}{ }_{0} F_{1}\left(l+2 ; a^{2} r^{2} / 4\right) C_{l}^{1}(\hat{\mathbf{a}} \cdot \hat{\mathbf{r}})=\sum_{l=0}^{\infty} \frac{a^{l}}{2^{l / 2} l !}{ }_{0} F_{1}\left(l+2 ; a^{2} r^{2} / 4\right)\left(C_{l}(\hat{\mathbf{a}}) \cdot\{\mathbf{r}\}_{l}\right) .
$$

This equation can be used for the derivation of the multipole decomposition for the scalar products $(\mathbf{a} \cdot \mathbf{r})^{n}$. Namely, one has to expand the exponent $\exp (\mathbf{a} \cdot \mathbf{r})$ and the hypergeometric 
function ${ }_{0} F_{1}\left(l+2 ; a^{2} r^{2} / 4\right)$ into the power series and compare the coefficients at equal powers of ar. This leads to the formula

$$
\begin{aligned}
(\mathbf{a} \cdot \mathbf{r})^{n}=n !\left(\frac{a r}{2}\right)^{n} \sum_{k=0}^{[n / 2]} \frac{n-2 k+1}{k !(n-k+1) !} C_{n-2 k}^{1}(\hat{\mathbf{a}} \cdot \hat{\mathbf{r}}) & \\
\quad=n !(a r)^{n} \sum_{l=n, n-2, \ldots} & \frac{2(l+1)}{(n-l) ! !(n+l+2) ! !}\left(C_{l}(\hat{\mathbf{a}}) \cdot C_{l}(\hat{\mathbf{r}})\right) .
\end{aligned}
$$

For small values of $n$ this formula has been checked explicitly.

Replacing in eq. (43) $\mathbf{r}$ with $\nabla$ one arrives at the following equation for the Taylor expansion (40),

$$
f(\mathbf{a}+\mathbf{r})=\sum_{l=0}^{\infty} \frac{a^{l}}{2^{l / 2} l !}{ }_{0} F_{1}\left(l+2 ; a^{2} \Delta / 4\right)\left(C_{l}(\hat{\mathbf{a}}) \cdot\{\nabla\}_{l}\right) f(\mathbf{r}) .
$$

This is the key equation for the derivation of multipole expansions.

For further consideration it is necessary to calculate the action of the Laplace operator on $\mathrm{HSH}$,

$$
\Delta C_{l}(\hat{\mathbf{r}})=\left(\frac{1}{r^{3}} \frac{\partial}{\partial r} r^{3} \frac{\partial}{\partial r}+\frac{\Delta_{\Omega}}{r^{2}}\right) C_{l}(\hat{\mathbf{r}})=\frac{\Delta_{\Omega}}{r^{2}} C_{l}(\hat{\mathbf{r}})=-\frac{l(l+2)}{r^{2}} C_{l}(\hat{\mathbf{r}}) .
$$

Hereafter, we will label HSH only with their ranks and, for the sake of shortness, we will omit all projection indices.

Using the above formula one can prove the identity

$$
\Delta f(r) C_{l}(\hat{\mathbf{r}})=C_{l}(\hat{\mathbf{r}})\left(\frac{1}{r^{3}} \frac{\partial}{\partial r} r^{3} \frac{\partial}{\partial r}-\frac{l(l+2)}{r^{2}}\right) f(r)
$$

where $f(r)$ is an arbitrary function depending on $r=|\mathbf{r}|$. This equation can be presented in two different compact forms

$$
\Delta f(r) C_{l}(\hat{\mathbf{r}})=C_{l}(\hat{\mathbf{r}}) \frac{1}{r^{l+3}} \frac{\partial}{\partial r} r^{2 l+3} \frac{\partial}{\partial r} \frac{1}{r^{l}} f(r)=C_{l}(\hat{\mathbf{r}}) r^{l-1} \frac{\partial}{\partial r} \frac{1}{r^{2 l+1}} \frac{\partial}{\partial r} r^{l+2} f(r) .
$$

In the important particular case of $f(r)=r^{n}$ these identities lead to the formula

$$
\Delta^{k} r^{n} C_{l}(\hat{\mathbf{r}})=C_{l}(\hat{\mathbf{r}}) r^{n-2 k} \frac{(n-l) ! !}{(n-l-2 k) ! !} \frac{(l+n+2) ! !}{(l+n+2-2 k) ! !} .
$$

One can re-write this equation in a form free of factorials

$$
\Delta^{k} r^{n} C_{l}(\hat{\mathbf{r}})=C_{l}(\hat{\mathbf{r}}) r^{n-2 k} 2^{2 k}\left(\frac{-2-l-n}{2}\right)_{k}\left(\frac{l-n}{2}\right)_{k}
$$

where $(a)_{k}=\Gamma(a+k) / \Gamma(a)$ is the Pochhammer symbol. As is seen, this equation is also valid in the case of non-integer values of $n$. 


\section{B. Multipole expansions of translated hyperspherical harmonics}

Below we calculate the multipole expansion of the function $f(\mathbf{a}+\mathbf{r})=|\mathbf{a}+\mathbf{r}|^{n} C_{j}(\hat{\mathbf{b}})$, where $\hat{\mathbf{b}}=(\mathbf{a}+\mathbf{r}) /|\mathbf{a}+\mathbf{r}|$. According to eq. (45), in order to calculate the multipole expansion of this function, one has to calculate the operator action

$$
\left(C_{l}(\hat{\mathbf{a}}) \cdot\{\nabla\}_{l}\right) f(\mathbf{r})=\left(C_{l}(\hat{\mathbf{a}}) \cdot\{\nabla\}_{l}\right) r^{n} C_{j}(\hat{\mathbf{r}}) .
$$

From the general symmetry arguments it is clear that this action can be presented as a combination of bipolar harmonics

$$
\left(C_{l}(\hat{\mathbf{a}}) \cdot\{\nabla\}_{l}\right) r^{n} C_{j}(\hat{\mathbf{r}})=r^{n-l} \sum_{l^{\prime}} A_{l l^{\prime}}\left\{C_{l}(\hat{\mathbf{a}}) \otimes C_{l^{\prime}}(\hat{\mathbf{r}})\right\}_{j},
$$

where the coefficients $A_{l l^{\prime}}$ are numbers which depend apart of $l, l^{\prime}$ also on $j$ and $n$ but are independent on vectors $\mathbf{a}$ and $\mathbf{r}$.

The independence of the coefficients $A_{l l^{\prime}}$ on vectors $\mathbf{a}$ and $\mathbf{r}$ allows one to simplify their calculation. We will calculate $A_{l l^{\prime}}$ for vectors a having zero length: $(\mathbf{a} \cdot \mathbf{a})=0$. Noting this fact and the expression (24) for the scalar product of $C$-harmonics we arrive at the identity

$$
\left(C_{l}(\hat{\mathbf{a}}) \cdot\{\nabla\}_{l}\right)=2^{l / 2}(\mathbf{a} \cdot \nabla)^{l} .
$$

Now one has to evaluate the action of the operator in the rhs of this equation on the product $r^{n} C_{j}(\hat{\mathbf{r}})$. This can be done using the chain differentiation rule

$$
(\mathbf{a} \cdot \nabla)^{l} r^{n} C_{j}(\hat{\mathbf{r}})=2^{j / 2}(\mathbf{a} \cdot \nabla)^{l} r^{n-j}\{\mathbf{r}\}_{j}=2^{j / 2} \sum_{k=0}^{l}\left(\begin{array}{l}
l \\
k
\end{array}\right)\left[(\mathbf{a} \cdot \nabla)^{k} r^{n-j}\right](\mathbf{a} \cdot \nabla)^{l-k}\{\mathbf{r}\}_{j},
$$

where $\left(\begin{array}{l}l \\ k\end{array}\right)=l ! /(k !(l-k) !)$ is the binomial coefficient and $\nabla$-operators in square brackets do not act on the outer terms. The term $(\mathbf{a} \cdot \nabla)^{l-k}\{\mathbf{r}\}_{j}$ can be evaluated using the vector differentiation technique described in [12] so that

$$
(\mathbf{a} \cdot \nabla)^{l-k}\{\mathbf{r}\}_{j}=\frac{j !}{(j-l+k) !}\left\{\{\mathbf{a}\}_{l-k} \otimes\{\mathbf{r}\}_{j-l+k}\right\}_{j} .
$$

The calculation of operator action in square brackets in (53) simplifies because $(\mathbf{a} \cdot \mathbf{a})=0$. After some simple analysis one obtains

$$
\begin{aligned}
(\mathbf{a} \cdot \nabla)^{k} r^{n-j}=(n-j)(n-j-2) \cdots(n-j-2 k & +2)(\mathbf{a} \cdot \mathbf{r})^{k} r^{n-j-2 k} \\
& =(-2)^{k}\left(-\frac{n-j}{2}\right)_{k}(\mathbf{a} \cdot \mathbf{r})^{k} r^{n-j-2 k}
\end{aligned}
$$


Now we have to re-write the combination $(\mathbf{a} \cdot \mathbf{r})^{k}\left\{\{\mathbf{a}\}_{l-k} \otimes\{\mathbf{r}\}_{j-l+k}\right\}_{j}$ in terms of HSH. This can be achieved using the tensor re-coupling rules. To illustrate this we note that the above construction can be presented in the form

$$
\left.(\mathbf{a} \cdot \mathbf{r})^{k}\left\{\{\mathbf{a}\}_{l-k} \otimes\{\mathbf{r}\}_{j-l+k}\right\}_{j}=r^{j-l+2 k}\left(\{\mathbf{a}\}_{k} \cdot\{\hat{\mathbf{r}}\}_{k}\right)\left\{\{\mathbf{a}\}_{l-k} \otimes \hat{\mathbf{r}}\right\}_{j-l+k}\right\}_{j} .
$$

The re-coupling of the tensor products in rhs of this equation yields

$$
\begin{aligned}
\left(\{\mathbf{a}\}_{k} \cdot\{\hat{\mathbf{r}}\}_{k}\right)\left\{\{\mathbf{a}\}_{l-k} \otimes\{\hat{\mathbf{r}}\}_{j-l+k}\right\}_{j}=2^{(l-j) / 2-k} & \sum_{l^{\prime}}(k+1)(j+1)(l+1)\left(l^{\prime}+1\right) \\
& \times\left[\begin{array}{ccc}
k & k & 0 \\
l-k & j-l+k & j \\
l & l^{\prime} & j
\end{array}\right]\left\{\{\hat{\mathbf{a}}\}_{l} \otimes C_{l^{\prime}}(\hat{\mathbf{r}})\right\}_{j},
\end{aligned}
$$

where we have used the auxiliary identity

$$
\left\{\{\mathbf{a}\}_{k} \otimes\{\mathbf{a}\}_{l-k}\right\}_{q}=\{\mathbf{a}\}_{l} \delta_{l q}
$$

which follows from the fact that $(\mathbf{a} \cdot \mathbf{a})=0$. The summation index $l^{\prime}$ in eq. (57) takes the values of $|j-l|,|j-l|+2, \ldots, j-l+2 k$. Thus, the combination $l+l^{\prime}+j$ is always an even number.

The four-dimensional re-coupling coefficient in eq. (57) is connected with threedimensional $9 j$-symbols by means of eq. (35). The resulting $9 j$-coefficient can be evaluated in closed form [1], so that the four-dimensional re-coupling coefficient becomes

$$
\begin{aligned}
& {\left[\begin{array}{ccr}
k & k & 0 \\
l-k & j-l+k & j \\
l & l^{\prime} & j
\end{array}\right]=(-1)^{j+l+l^{\prime}} \frac{k !(j-l+k) !}{(l+1) !(j+1) !(k+1)(j+1)}} \\
& \times \frac{\Gamma\left(\frac{j+l+l^{\prime}}{2}+2\right) \Gamma\left(\frac{j+l-l^{\prime}}{2}+1\right)}{\Gamma\left(\frac{j-l-l^{\prime}}{2}+k+1\right) \Gamma\left(\frac{j-l+l^{\prime}}{2}+k+2\right)} .
\end{aligned}
$$

The next step is to insert this equation into eq. (57) and substitute the result together with eq. (55) into eq. (53). This leads to the identity

$$
\begin{aligned}
(\mathbf{a} \cdot \nabla)^{l} r^{n} C_{j}(\hat{\mathbf{r}})=r^{n-l} & 2^{l / 2} \sum_{l^{\prime}}\left\{\{\mathbf{a}\}_{l} \otimes C_{l^{\prime}}(\hat{\mathbf{r}})\right\}_{j}(-1)^{j+l+l^{\prime}} \frac{l^{\prime}+1}{j+1} \\
& \times \sum_{k=0}^{l} \frac{(-1)^{k}}{(l-k) !}\left(\frac{j-n}{2}\right)_{k} \frac{\Gamma\left(\frac{j+l+l^{\prime}}{2}+2\right) \Gamma\left(\frac{j+l-l^{\prime}}{2}+1\right)}{\Gamma\left(\frac{j-l-l^{\prime}}{2}+k+1\right) \Gamma\left(\frac{j-l+l^{\prime}}{2}+k+2\right)} .
\end{aligned}
$$


Here, the summation over $k$ can be performed analytically. Noting also the identity (52) the above equation evaluates to

$$
\begin{aligned}
\left(C_{l}(\hat{\mathbf{a}}) \cdot\{\nabla\}_{l}\right) r^{n} C_{j}(\hat{\mathbf{r}})=r^{n-l} 2^{l / 2} \sum_{l^{\prime}}(-1)^{l}\left\{C_{l}(\hat{\mathbf{a}}) \otimes C_{l^{\prime}}(\hat{\mathbf{r}})\right\}_{j} \frac{l^{\prime}+1}{j+1} & \\
& \times\left(\frac{-2-j-n}{2}\right)_{\left(j+l-l^{\prime}\right) / 2}\left(\frac{j-n}{2}\right)_{\left(l+l^{\prime}-j\right) / 2},
\end{aligned}
$$

where we have used the fact that $(-1)^{j+l+l^{\prime}}=1$. It is important to note that the derived equation is valid for arbitrary vectors a (i.e. not only for zero-length vectors).

According to eq. (45), we have to act on eq. (60) with the operator ${ }_{0} F_{1}\left(l+2 ; a^{2} \Delta / 4\right)$. Thus, one has to calculate the operator construction

$$
{ }_{0} F_{1}\left(l+2 ; a^{2} \Delta / 4\right) r^{n-l} C_{l^{\prime}}(\hat{\mathbf{r}})=\sum_{k=0}^{\infty} \frac{a^{2 k}}{2^{2 k} k !(l+2)_{k}} \Delta^{k} r^{n-l} C_{l^{\prime}}(\hat{\mathbf{r}}) .
$$

Equation (50) allows one to calculate the action of Laplace operators $\Delta^{k}$ on the product $r^{n-l} C_{l^{\prime}}(\hat{\mathbf{r}})$. The resulting series leads to the Gauss hypergeometric function,

$$
\begin{aligned}
C_{l^{\prime}}(\hat{\mathbf{r}}) \sum_{k=0}^{\infty} \frac{r^{n-l-2 k} a^{2 k}}{k !(l+2)_{k}}\left(\frac{-2+l-l^{\prime}-n}{2}\right)_{k}\left(\frac{l+l^{\prime}-n}{2}\right)_{k} \\
=r^{n-l} C_{l^{\prime}}(\hat{\mathbf{r}})_{2} F_{1}\left(\frac{-2+l-l^{\prime}-n}{2}, \frac{l+l^{\prime}-n}{2} ; l+2 ; \frac{a^{2}}{r^{2}}\right) .
\end{aligned}
$$

For the sake of simpler presentation it is convenient to replace the notations by $\mathbf{a} \rightarrow \mathbf{r}_{1}$ and $\mathbf{r} \rightarrow \mathbf{r}_{2}$. Now we can write the explicit expression for the multipole expansion of the product $r^{n} C_{j}(\hat{\mathbf{r}})$, where $\mathbf{r}=\mathbf{r}_{1}+\mathbf{r}_{2}$,

$$
\begin{aligned}
r^{n} C_{j}(\hat{\mathbf{r}})= & \sum_{l, l^{\prime}=0}^{\infty} B_{l l^{\prime}}^{(n j)}\left(r_{1}, r_{2}\right)\left\{C_{l}\left(\hat{\mathbf{r}}_{1}\right) \otimes C_{l^{\prime}}\left(\hat{\mathbf{r}}_{2}\right)\right\}_{j}, \\
B_{l l^{\prime}}^{(n j)}\left(r_{1}, r_{2}\right)= & r_{2}^{n}\left(-\frac{r_{1}}{r_{2}}\right)^{l} \frac{l^{\prime}+1}{l !(j+1)}\left(\frac{-2-j-n}{2}\right)_{\left(j+l-l^{\prime}\right) / 2}\left(\frac{j-n}{2}\right)_{\left(l+l^{\prime}-j\right) / 2} \\
& \times{ }_{2} F_{1}\left(\frac{-2+l-l^{\prime}-n}{2}, \frac{l+l^{\prime}-n}{2} ; l+2 ; \frac{r_{1}^{2}}{r_{2}^{2}}\right),
\end{aligned}
$$

where the summations over $l, l^{\prime}$ are performed over all values at which $j+l-l^{\prime}=0,2,4, \ldots$ and $l+l^{\prime} \geq j$. Thus, there is only one infinite summation in the above formula.

Since the combination $\left(l+l^{\prime}-j\right) / 2$ is a positive integer number, at negative integer values of $(j-n) / 2$ the above multipole series are, in fact, finite sums. Indeed, for $(n-j)=2,4, \ldots$, 
the second Pochhammer symbols in the expression for $B_{l l^{\prime}}^{(n j)}$ vanishes at all values of $l, l^{\prime}$ except those with $\left(l+l^{\prime}-j\right) \leq|j-n|$.

We note also that for $r_{1}=i, r_{2}=1$ and $j=0, n=2 N$, where $N$ can be arbitrary integer, the lhs of eq. (65) reduces to

$$
\left(2 i\left(\hat{\mathbf{r}}_{1} \cdot \hat{\mathbf{r}}_{2}\right)\right)^{N}=\sum_{l=N, N-2, \ldots} B_{l l}^{(2 N, 0)}(i, 1)\left\{C_{l}\left(\hat{\mathbf{r}}_{1}\right) \otimes C_{l}\left(\hat{\mathbf{r}}_{2}\right)\right\}_{0} .
$$

Thus, it is the multipole expansion for the powers of a scalar product which has been derived above in a different way, see eq. (44).

For finite multipole decompositions the question of convergence does not occur. The infinite multipole series is convergent only when $r_{1}<r_{2}$. In the opposite case (i.e. at $r_{1}>r_{2}$ ) the replacement $\mathbf{r}_{1} \leftrightarrow \mathbf{r}_{2}$ recovers the convergence of the corresponding series.

Below we present several explicit examples of the multipole expansion (63). There are two situations when they have particularly simple form. Namely, at $n=j$ or $n=-j-2$ the product $r^{n} C_{j}(\hat{\mathbf{r}})$ satisfies the Laplace equation

$$
\Delta r^{j} C_{j}(\hat{\mathbf{r}})=\Delta \frac{1}{r^{j+2}} C_{j}(\hat{\mathbf{r}})=0 .
$$

As a consequence, the Gauss hypergeometric function in (63) is equal to unity. This is clearly seen also from eq. (61). The corresponding multipole expansions are

$$
\begin{aligned}
r^{j} C_{j}(\hat{\mathbf{r}}) & =\sum_{l=0}^{j}\left(\begin{array}{l}
j \\
l
\end{array}\right) r_{1}^{l} r_{2}^{j-l}\left\{C_{l}\left(\hat{\mathbf{r}}_{1}\right) \otimes C_{j-l}\left(\hat{\mathbf{r}}_{2}\right)\right\}_{j}, \\
\frac{1}{r^{j+2}} C_{j}(\hat{\mathbf{r}}) & =\sum_{l=0}^{\infty}(-1)^{l} \frac{r_{1}^{l}}{r_{2}^{j+l+2}}\left(\begin{array}{c}
j+l+1 \\
l
\end{array}\right)\left\{C_{l}\left(\hat{\mathbf{r}}_{1}\right) \otimes C_{j+l}\left(\hat{\mathbf{r}}_{2}\right)\right\}_{j},
\end{aligned}
$$

where $\mathbf{r}=\mathbf{r}_{1}+\mathbf{r}_{2}$.

Let us consider one more example of the multipole expansion eq. (63). At $j=0$ we have to expand the scalar function $\left|\mathbf{r}_{1}+\mathbf{r}_{2}\right|^{n}$. In this case $l=l^{\prime}$, and noting the identity

$$
\left\{C_{l}\left(\hat{\mathbf{r}}_{1}\right) \otimes C_{l}\left(\hat{\mathbf{r}}_{2}\right)\right\}_{0}=\frac{1}{l+1}\left(C_{l}\left(\hat{\mathbf{r}}_{1}\right) \cdot C_{l}\left(\hat{\mathbf{r}}_{2}\right)\right)
$$

we can write the multipole decomposition of the function $\left|\mathbf{r}_{1}+\mathbf{r}_{2}\right|^{n}$ as

$$
\begin{aligned}
\left|\mathbf{r}_{1}+\mathbf{r}_{2}\right|^{n}=r_{2}^{n} \sum_{l=0}^{\infty}\left(-\frac{r_{1}}{r_{2}}\right)^{l} \frac{1}{l !}\left(-\frac{n}{2}\right)_{l}{ }_{l} F_{1}\left(-1-\frac{n}{2}, l-\frac{n}{2} ; l+2 ;\right. & \left.\frac{r_{1}^{2}}{r_{2}^{2}}\right) \\
& \times\left(C_{l}\left(\hat{\mathbf{r}}_{1}\right) \cdot C_{l}\left(\hat{\mathbf{r}}_{2}\right)\right) .
\end{aligned}
$$


We note that for $n$ being an even integer number, the Gauss hypergeometric function reduces to the associated Legendre polynomial. As is seen, the derived multipole series are convergent only for $r_{1}<r_{2}$. In the opposite case $r_{1}>r_{2}$, the replacement $\mathbf{r}_{1} \leftrightarrow \mathbf{r}_{2}$ should be made in the above formula.

\section{CONCLUSION}

The main results of this paper are the multipole expansions given by eqs. (44), (63), (64), (65) and (67). The derivations given in the paper can be directly generalised on the case of space with dimensionality larger than four. According to eq. (57), this requires the knowledge of corresponding re-coupling coefficients. Since the four-dimensional HSH are proportional to the wave functions of the Hydrogen atom, various integrals involving those functions can be calculated using the technique of multipole expansions developed above. Examples of such calculations will be reported in a forthcoming publication.

Finally, we note that eq. (63) can be used for the derivation of the general multipole expansion of the function $f(r) C_{j}(\hat{\mathbf{r}})$ where $\mathbf{r}=\mathbf{r}_{1}+\mathbf{r}_{2}$. Namely, we write the Taylor series for the function $f(r)$,

$$
f(r) C_{j}(\hat{\mathbf{r}})=\sum_{n=0}^{\infty} f_{n} r^{n} C_{j}(\hat{\mathbf{r}}) .
$$

Each term of this equation can be decomposed using eq. (63), which leads to the equation

$$
f(r) C_{j}(\hat{\mathbf{r}})=\sum_{l, l^{\prime}} C_{l l^{\prime}}^{(j)}\left(r_{1}, r_{2}\right)\left\{C_{l}\left(\hat{\mathbf{r}}_{1}\right) \otimes C_{l^{\prime}}\left(\hat{\mathbf{r}}_{2}\right)\right\}_{j}
$$

where the coefficients $C_{l l^{\prime}}^{(j)}$ are defined by

$$
C_{l l^{\prime}}^{(j)}\left(r_{1}, r_{2}\right)=\sum_{n=0}^{\infty} f_{n} B_{l l^{\prime}}^{(n j)}\left(r_{1}, r_{2}\right)
$$

The expressions for the parameters $B_{l l^{\prime}}^{(n j)}\left(r_{1}, r_{2}\right)$ are given by eq. (63).

\section{Acknowledgments}

This work has been supported in part by the BRHE joint program of CRDF and Russian ministry of education under the grant No. Y2-CP-10-02. I am grateful to Jan-Michael Rost for careful reading of the manuscript and providing useful suggestions. 


\section{APPENDIX A: EXPRESSIONS FOR FOUR-DIMENSIONAL CLEBSCH-} GORDAN COEFFICIENTS

The expression for $C$-type CGC simplifies for $j=j_{1}+j_{2}$. In this case the $9 j$-symbol in the definition (27) of $C$-type CGC can be re-written in terms of $3 d$ CGC, see eq. (9) of Sec. 10.8.3 of [1]. As a result, one arrives at the equation

$$
\begin{aligned}
C_{j_{1} \lambda_{1} \alpha_{1} ; j_{2} \lambda_{2} \alpha_{2}}^{\left(j_{1}+j_{2}\right) \lambda \alpha}= & C_{\lambda_{1} 0 \lambda_{2} 0}^{\lambda 0} C_{\lambda_{1} \alpha_{1} \lambda_{2} \alpha_{2}}^{\lambda \alpha} \frac{j_{1} ! j_{2} !}{\left(j_{1}+j_{2}\right) !} \\
& \times\left[\frac{\left(j_{1}+j_{2}+\lambda+1\right) !\left(j_{1}+j_{2}-\lambda\right) !\left(2 \lambda_{1}+1\right)\left(2 \lambda_{2}+1\right)}{\left(j_{1}+\lambda_{1}+1\right) !\left(j_{1}-\lambda_{1}\right) !\left(j_{2}+\lambda_{2}+1\right) !\left(j_{2}-\lambda_{2}\right) !(2 \lambda+1)}\right]^{1 / 2} .
\end{aligned}
$$

This equation simplifies significantly for $\lambda_{1}=\alpha_{1}=0$. In this case the three-dimensional CGC are equal to unity, so that

$$
C_{j_{1} 00 ; j_{2} \lambda_{2} \alpha_{2}}^{\left(j_{1}+j_{2}\right) \lambda \alpha}=\delta_{\lambda, \lambda_{2}} \delta_{\alpha, \alpha_{2}} \frac{j_{2} !}{\left(j_{1}+j_{2}\right) !}\left[\frac{\left(j_{1}+j_{2}+\lambda+1\right) !\left(j_{1}+j_{2}-\lambda\right) !}{\left(j_{1}+1\right)\left(j_{2}+\lambda+1\right) !\left(j_{2}-\lambda\right) !}\right]^{1 / 2}
$$

For $\lambda=\alpha=0$ this equation reduces to

$$
C_{j_{1} 00 ; j_{2} 00}^{\left(j_{1}+j_{2}\right) 00}=\left[\frac{j_{1}+j_{2}+1}{\left(j_{1}+1\right)\left(j_{2}+1\right)}\right]^{1 / 2}
$$

which is in agreement with the general equation (33). We consider also the particular case of eq. (A1) for maximal values of projections $\lambda$,

$$
C_{j_{1} j_{1} \alpha_{1} ; j_{2} j_{2} \alpha_{2}}^{\left(j_{1}+j_{2}\right)\left(j_{1}+j_{2}\right) \alpha}=C_{j_{1} \alpha_{1} j_{2} \alpha_{2}}^{\left(j_{1}+j_{2}\right) \alpha}
$$

For maximal possible values of both $\lambda$ - and $\alpha$-projections one has

$$
C_{j_{1} j_{1} j_{1} ; j_{2} j_{2} j_{2}}^{\left(j_{1}+j_{2}\right)\left(j_{1}+j_{2}\right)}=1
$$

We present also the expression for CGC with $j=j_{2}-j_{1}$, which can be derived from (A1) and the symmetry relations (30) and (31),

$$
\begin{aligned}
C_{j_{1} \lambda_{1} \alpha_{1} ; j_{2} \lambda_{2} \alpha_{2}}^{\left(j_{2}-j_{1}\right) \lambda \alpha}=C_{\lambda 0 \lambda_{1} 0}^{\lambda_{2} 0} C_{\lambda_{1} \alpha_{1} \lambda_{2} \alpha_{2}}^{\lambda \alpha} \frac{j_{1} !\left(j_{2}-j_{1}+1\right) !}{\left(j_{2}+1\right) !} & \\
& \times\left[\frac{\left(j_{2}+\lambda_{2}+1\right) !\left(j_{2}-\lambda_{2}\right) !\left(2 \lambda_{1}+1\right)}{\left(j_{1}+\lambda_{1}+1\right) !\left(j_{1}-\lambda_{1}\right) !\left(j_{2}-j_{1}+\lambda+1\right) !\left(j_{2}-j_{1}-\lambda\right) !}\right]^{1 / 2} .
\end{aligned}
$$

It is also of interest to consider CGC with some set of projections equal to zero, e.g. $\lambda_{1}=\alpha_{1}=0$. In this case, $\lambda=\lambda_{2}$ and $\alpha=\alpha_{2}$ and the $9 j$-coefficient in the definition (27) of 
CGC reduces to the $6 j$-coefficient [1],

$$
C_{j_{1} 00 ; j_{2} \lambda \alpha}^{j \lambda \alpha}=(-1)^{\lambda+\left(j_{1}+j_{2}+j\right) / 2} \frac{j+1}{\sqrt{j_{1}+1}}\left\{\begin{array}{ccc}
\lambda & \frac{j}{2} & \frac{j}{2} \\
\frac{j_{1}}{2} & \frac{j_{2}}{2} & \frac{j_{2}}{2}
\end{array}\right\} .
$$

In particular, for $j_{1}=1$ we have that $j=j_{2} \pm 1$ and evaluating the $6 j$-symbol to its explicit form we obtain

$$
C_{100 ; j \lambda \alpha}^{(j-1) \lambda \alpha}=\frac{\sqrt{(j-\lambda)(j+\lambda+1)}}{(j+1) \sqrt{2}}, \quad C_{100 ; j \lambda \alpha}^{(j+1) \lambda \alpha}=\frac{\sqrt{(j-\lambda+1)(j+\lambda+2)}}{(j+1) \sqrt{2}} .
$$

[1] D. A. Varshalovich, A. N. Moskalev, and V. K. Khersonskii, Quantum theory of angular momentum (World Scientific, Singapore, 1988).

[2] R. A. Sack, J. Math. Phys. 5, 252 (1964).

[3] Z.-Y. Wen and J. Avery, J. Math. Phys. 26, 396 (1985).

[4] N. L. Manakov, A. V. Meremianin, and A. F. Starace, J. Phys. B: At. Mol. Opt. Phys. 35, 77 (2002).

[5] W. Fock, Z. Phys. 98, 145 (1935).

[6] J. Avery, Int. J. Quantum Chem. 100, 121 (2004).

[7] V. Aquilanti, S. Cavalli, C. Coletti, D. di Domenico, and G. Grossi, Int. Reviews in Physical Chemistry 20, 673 (2001).

[8] V. Aquilanti, S. Cavalli, and C. Coletti, Phys. Rev. Lett. 80, 3209 (1998).

[9] A. Z. Dolginov and I. N. Toptygin, Sov. Phys.-JETP 8, 550 (1959).

[10] L. C. Biedenharn, J. Math. Phys. 2, 433 (1960).

[11] A. Erdelyi, W. Magnus, F. Oberhettinger, and F. G. Tricomi, Higher trancendental functions. Bateman manuscript project, vol. II (McGraw-hill book company, Inc, 1953).

[12] N. L. Manakov, S. I. Marmo, and A. V. Meremianin, J. Phys. B: At. Mol. Opt. Phys. 29, $2711(1996)$. 